\title{
DYSON'S CRANK OF A PARTITION
}

\author{
GEORGE E. ANDREWS AND F. G. GARVAN
}

1. Introduction. In [3], F. J. Dyson defined the rank of a partition as the largest part minus the number of parts. He let $N(m, t, n)$ denote the number of partitions of $n$ of rank congruent to $m$ modulo $t$, and he conjectured

$$
\begin{array}{ll}
N(m, 5,5 n+4)=\frac{1}{5} p(5 n+4), & 0 \leq m \leq 4 ; \\
N(m, 7,7 n+5)=\frac{1}{7} p(7 n+5), & 0 \leq m \leq 6,
\end{array}
$$

where $p(n)$ is the total number of partitions of $n[1$, Chapter 1]. These conjectures were subsequently proved by Atkin and Swinnerton-Dyer [2].

Dyson [3] went on to observe that the rank did not separate the partition of $11 n+6$ into 11 equal classes even though Ramanujan's congruence

$$
p(11 n+6) \equiv 0 \quad(\bmod 11)
$$

holds. He was thus led to conjecture the existence of some other partition statistic (which he called the crank); this unknown statistic should provide a combinatorial interpretation of $\frac{1}{11} p(11 n+6)$ in the same way that $(1.1)$ and (1.2) treat the primes 5 and 7.

In $[\mathbf{4}, \mathbf{5}]$, one of us was able to find a crank relative to vector partitions as follows:

For a partition $\pi$, let $\#(\pi)$ be the number of parts of $\pi$ and $\sigma(\pi)$ be the sum of the parts of $\pi$ (or the number $\pi$ is partitioning) with the convention $\#(\phi)=\sigma(\phi)=0$ for the empty partition $\phi$, of 0 . Let

$$
V=\left\{\left(\pi_{1}, \pi_{2}, \pi_{3}\right) \mid \pi_{1}\right. \text { is a partition into distinct parts, }
$$

$$
\left.\pi_{2}, \pi_{3} \text { are unrestricted partitions }\right\} \text {. }
$$

We shall call the elements of $V$ vector partitions. For $\vec{\pi}=\left(\pi_{1}, \pi_{2}, \pi_{3}\right)$ in $V$ we define the sum of parts, $s$, a weight, $\omega$, and a crank, $r$, by

$$
\begin{gathered}
s(\vec{\pi})=\sigma\left(\pi_{1}\right)+\sigma\left(\pi_{2}\right)+\sigma\left(\pi_{3}\right), \\
\omega(\vec{\pi})=(-1)^{\#\left(\pi_{1}\right)}, \\
r(\vec{\pi})=\#\left(\pi_{2}\right)-\#\left(\pi_{3}\right) .
\end{gathered}
$$

We say $\vec{\pi}$ is a vector partition of $n$ if $s(\vec{\pi})=n$. For example, if

$$
\vec{\pi}=(5+3+2,2+2+1,2+1+1)
$$

Received by the editors August 13, 1987.

1980 Mathematics Subject Classification (1985 Revision). Primary 11 P76.

First author partially supported by National Science Foundation Grant DMS 8503324. 
then $s(\vec{\pi})=19, \omega(\vec{\pi})=-1, r(\vec{\pi})=0$ and $\vec{\pi}$ is a vector partition of 19 . The number of vector partitions of $n$ (counted according to the weight $\omega$ ) with crank $m$ is denoted by $N_{V}(m, n)$, so that

$$
N_{V}(m, n)=\sum_{\substack{\vec{\pi} \in V \\ s(\vec{\pi})=n \\ r(\vec{\pi})=m}} \omega(\vec{\pi})
$$

The number of vector partitions of $n$ (counted according to the weight $\omega$ ) with crank congruent to $k$ modulo $t$ is denoted by $N_{V}(k, t, n)$, so that

$$
N_{V}(k, t, n)=\sum_{m=-\infty}^{\infty} N_{V}(m t+k, n)=\sum_{\substack{\vec{\pi} \in V \\ s(\vec{\pi})=n \\ r(\vec{\pi}) \equiv k(\bmod t)}} \omega(\vec{\pi}) .
$$

By considering the transformation that interchanges $\pi_{2}$ and $\pi_{3}$ we have

$$
N_{V}(m, n)=N_{V}(-m, n)
$$

so that

$$
N_{V}(t-m, t, n)=N_{V}(m, t, n) .
$$

We have the following generating function for $N_{V}(m, n)$ :

$$
\sum_{m=-\infty}^{\infty} \sum_{n=0}^{\infty} N_{V}(m, n) z^{m} q^{n}=\prod_{n=1}^{\infty} \frac{\left(1-q^{n}\right)}{\left(1-z q^{n}\right)\left(1-z^{-1} q^{n}\right)}
$$

By putting $z=1$ in (1.11) we find

$$
\sum_{m=-\infty}^{\infty} N_{V}(m, n)=p(n)
$$

VECTOR-CRANK THEOREM (GARVAN $[\mathbf{4}, \mathbf{5}]$ ).

$$
N_{V}(0,5,5 n+4)=N_{V}(1,5,5 n+4)=\cdots=N_{V}(4,5,5 n+4)=\frac{p(5 n+4)}{5},
$$

$$
\begin{aligned}
& N_{V}(0,7,7 n+5)=N_{V}(1,7,7 n+5)=\cdots=N_{V}(6,7,7 n+5)=\frac{p(7 n+5)}{7}, \\
& \text { 1.15) } \quad N_{V}(0,11,11 n+6)=\cdots=N_{V}(10,11,11 n+6)=\frac{p(11 n+6)}{11} .
\end{aligned}
$$

The above still leaves open the question of whether there is a crank for ordinary partitions. The answer is "yes" when the crank is defined as follows:

DEFINITION. For a partition $\pi$, let $l(\pi)$ denote the largest part of $\pi, \omega(\pi)$ denote the number of ones in $\pi$, and $\mu(\pi)$ denote the number of parts of $\pi$ larger than $\omega(\pi)$. The crank $c(\pi)$ is given by

$$
c(\pi)= \begin{cases}l(\pi) & \text { if } \omega(\pi)=0, \\ \mu(\pi)-\omega(\pi) & \text { if } \omega(\pi)>0 .\end{cases}
$$


Our main result is the following.

THEOREM 1. The number of partitions $\pi$ of $n$ with $c(\pi)=m$ is $N_{V}(m, n)$ for all $n>1$.

Obviously, in light of the Vector-Crank Theorem, we see that Theorem 1 supplies the crank asked for by Dyson.

2. PROOF OF THEOREM 1 . We shall require the standard notation of $q$-series:

$$
(A ; q)_{n}=(A)_{n}=\prod_{j=0}^{\infty} \frac{\left(1-A q^{j}\right)}{\left(1-A q^{j+n}\right)}
$$

$\left(=(1-A)(1-A q) \cdots\left(1-A q^{n-1}\right)\right.$ when $n$ is a positive integer $)$, and

$$
(A ; q)_{\infty}=(A)_{\infty}=\prod_{j=0}^{\infty}\left(1-A q^{j}\right)
$$

We now transform (1.11):

$$
\begin{aligned}
\sum_{m=-\infty}^{\infty} \sum_{n=0}^{\infty} N_{V}(m, n) z^{m} q^{n} & =\frac{(1-q)}{(z q)_{\infty}} \cdot \frac{\left(q^{2} ; q\right)_{\infty}}{(q / z)_{\infty}} \\
& =\frac{(1-q)}{(z q)_{\infty}} \sum_{j=0}^{\infty} \frac{(z q)_{j}(q / z)^{j}}{(q)_{j}} \quad \text { by [1, p. 17]) } \\
& =\frac{(1-q)}{(z q)_{\infty}}+\sum_{j=1}^{\infty} \frac{q^{j} z^{-j}}{\left(q^{2} ; q\right)_{j-1}\left(z q^{j+1}\right)_{\infty}}
\end{aligned}
$$

As was noted in (1.12), when we set $z=1$ the series on the left of (2.3) reduces to the generating function for $p(n)$. For $j>0$, the $j$ th term in the sum on the right is

$$
\frac{z^{-j} q^{\overbrace{1+1+\cdots+1}^{j \text { times }}}}{\left(1-q^{2}\right)\left(1-q^{3}\right) \cdots\left(1-q^{j}\right)\left(1-z q^{j+1}\right)\left(1-z q^{j+2}\right) \cdots} .
$$

The standard techniques of partition theory [1, Chapter 1] show that this expression generates partitions with $\omega(\pi)=j$ and the exponent on $z$ is clearly $\mu(\pi)-\omega(\pi)$, i.e. $c(\pi)$, since $j>0$.

Thus we must interpret

$$
\frac{(1-q)}{(1-z q)\left(1-z q^{2}\right)\left(1-z q^{3}\right) \cdots}
$$

as the generating function for partitions without ones. By considering conjugate partitions, we note that

$$
\frac{1}{(1-z q)\left(1-z q^{2}\right)\left(1-z q^{3}\right) \cdots}
$$


generates all partitions with the exponent on $z$ counting the largest part, and for integers larger than 1

$$
\frac{q}{(1-z q)\left(1-z q^{2}\right)\left(1-z q^{3}\right) \cdots}
$$

generates partitions with at least one 1 appearing again with the exponent on $z$ counting the largest part. Note that this interpretation fails for 1 because this is the unique instance in which introducing a 1 into the partitions of $n-1$ alters the largest part. Hence

$$
\frac{1-q}{(z q)_{\infty}}
$$

counts (for $n>1$ ) the number of partitions with no ones and with the exponent on $z$ being the largest part of the partition $l(\pi)=c(\pi)$. Thus in the double series expansion of

$$
\frac{(1-q)}{(z q)_{\infty}}+\sum_{j=1}^{\infty} \frac{q^{j} z^{-j}}{\left(q^{2} ; q\right)_{j-1}\left(z q^{j+1}\right)_{\infty}}
$$

we see that the coefficient of $z^{m} q^{n}(n>1)$ is the number of ordinary partitions of $n$ in which $c(\pi)=m$. Therefore by (2.3), we have Theorem 1 .

3. Conclusion. We can't resist exhibiting $c(\pi)$ for the first instance of (1.3).

\begin{tabular}{crrrr} 
partitions of 6 & $l(\pi)$ & $\omega(\pi)$ & $\mu(\pi)$ & $c(\pi)$ \\
\hline 6 & 6 & 0 & 1 & 6 \\
$5+1$ & 5 & 1 & 1 & 0 \\
$4+2$ & 4 & 0 & 2 & 4 \\
$4+1+1$ & 4 & 2 & 1 & -1 \\
$3+3$ & 3 & 0 & 2 & 3 \\
$3+2+1$ & 3 & 1 & 2 & 1 \\
$3+1+1+1$ & 3 & 3 & 0 & -3 \\
$2+2+2$ & 2 & 0 & 3 & 2 \\
$2+2+1+1$ & 2 & 2 & 0 & -2 \\
$2+1+1+1+1$ & 2 & 4 & 0 & -4 \\
$1+1+1+1+1+1$ & 1 & 6 & 0 & -6
\end{tabular}

As Theorem 1 together with (1.15) predicts, $c(\pi)$ provides eleven different residue classes modulo 11 .

\section{REFERENCES}

1. G. E. Andrews, The theory of partitions, Encyclopedia of Mathematics and Its Applications, Vol. 2 (G.-C. Rota, ed.), Addison-Wesley, Reading, 1976 (Reprinted: Cambridge Univ. Press, London and New York, 1984).

2. A. O. L. Atkin and P. Swinnerton-Dyer, Some properties of partitions, Proc. London Math. Soc. (3) 4 (1954), 84-106.

3. F. J. Dyson, Some guesses in the theory of partitions, Eureka (Cambridge) 8 (1944), 10-15.

4. F. G. Garvan, Generalizations of Dyson's rank, Ph. D. thesis, Pennsylvania State Univ., 1986. 
5. _ New combinatorial interpretations of Ramanujan's partition congruences mod 5, 7 and 11, Trans. Amer. Math. Soc. (to appear).

Department of Mathematics, Pennsylvania State University, UniverSITY PARK, PENNSYlVANIA 16802

DEPARTMENT OF MATHEMATICS, UNIVERSITY OF WISCONSIN-MADISON, MADISON, WISCONSIN 53706

Current address (F. G. Garvan): Institute for Mathematics and its Applications, University of Minnesota, Minneapolis, Minnesota 55455 
